The Astrophysical Journal, 686:000-000, 2008 October 10

(C) 2008. The American Astronomical Society. All rights reserved. Printed in U.S.A.

\title{
INTERMEDIATE-MASS BLACK HOLE INDUCED QUENCHING OF MASS SEGREGATION IN STAR CLUSTERS
}

\author{
Michael Gill, ${ }^{1}$ Michele Trenti, ${ }^{2}$ M. Coleman Miller, ${ }^{1}$ Roeland van der Marel, ${ }^{2}$ \\ Douglas Hamilton, ${ }^{1}$ and Massimo Stiavelli ${ }^{2}$ \\ Received 2008 May 13; accepted 2008 June 22
}

\begin{abstract}
In many theoretical scenarios it is expected that intermediate-mass black holes (IMBHs, with masses $M \sim 10^{2}-$ $10^{4} M_{\odot}$ ) reside at the centers of some globular clusters. However, observational evidence for their existence is limited. Several previous numerical investigations have focused on the impact of an IMBH on the cluster dynamics or brightness profile. Here we instead present results from a large set of direct $N$-body simulations including single and binary stars. These show that there is a potentially more detectable IMBH signature, namely on the variation of the average stellar mass between the center and the half-light radius. We find that the existence of an IMBH quenches mass segregation and causes the average mass to exhibit only modest radial variation in collisionally relaxed star clusters. This differs from when there is no IMBH. To measure this observationally requires high-resolution imaging at the level of that already available from the Hubble Space Telescope (HST) for the cores of a large sample of galactic globular clusters. With a modest additional investment of $H S T$ time to acquire fields around the half-light radius, it will be possible to identify the best candidate clusters to harbor an IMBH. This test can be applied only to globulars with a half-light relaxation time $\lesssim 1 \mathrm{Gyr}$, which is required to guarantee efficient energy equipartition due to twobody relaxation.
\end{abstract}

Subject headings: globular clusters: general — methods: $n$-body simulations — stellar dynamics

\section{INTRODUCTION}

Theoretical work has suggested that some globular clusters may harbor intermediate-mass black holes (IMBHs; $M \sim 10^{2}-$ $10^{4} M_{\odot}$ ) in their centers (e.g., Portegies Zwart et al. 2004). If this is indeed the case, there are significant consequences for ultraluminous X-ray sources, gravitational wave emission from dense star clusters, and the dynamics of globular clusters (GCs) in general (see van der Marel [2004] and Miller \& Colbert [2004] for an overview). Definitive evidence for IMBHs has, however, been elusive. For example, Gebhardt et al. $(2002,2005)$ argued for an IMBH in G1 based on the analysis of HST line-of-sight velocity data and Keck spectra, but an alternative analysis by Baumgardt et al. (2003a) points out that acceptable dynamic models without a large central object also fit the observations. Gerssen et al. $(2002,2003)$ argued that the kinematics of M15 seem to slightly favor the presence of an IMBH, but for this cluster alternative interpretations exist (Baumgardt et al. 2003b; Dull et al. 2003). More recently, the observed line-of-sight kinematics of $\omega$ Cen have also been used to argue for the presence of an IMBH (Noyola et al. 2008).

A more secure identification of an IMBH in a GC can, in principle, be provided also by measuring the proper motion of central stars in order to reconstruct their orbits and thus firmly establish if a central massive point object is present. Several HST-GO programs based on this idea have been approved in past cycles (e.g., GO10474, PI: Drukier; GO10401 and GO10841, PI: Chandar; GTO/ACS10335, PI: Ford), but to date they have not yielded any indisputable detections. The limitation for such studies is the need to carry out multiyear observations; thus prog-

\footnotetext{
${ }^{1}$ Department of Astronomy, University of Maryland, College Park, MD 20742-2421.

2 Space Telescope Science Institute, 3700 San Martin Drive, Baltimore, MD 21218.
}

ress is slow. To maximize the chances of success it is thus of primary importance to focus the observations on the candidates most likely to harbor an IMBH.

Candidate selection is possible if one focuses on the indirect influence of the IMBH on the dynamics of its host. Direct $N$-body simulations by Baumgardt et al. (2004) and Trenti et al. (2007a) found that the presence of an IMBH acts as a central energy source that is able to prevent gravothermal collapse and thus maintain a sizable core to half-mass radius ratio throughout the entire life of the GC. The existence of such a large $(\gtrsim 0.1)$ core to half-mass radius ratio in a collisionally relaxed cluster might be due to the presence of an IMBH (see also Heggie et al. 2007). However, the picture becomes more complicated when this signature is transferred from the ideal world of $N$-body simulations, where a complete knowledge of the system is available, to real observations, where essentially only main-sequence and red giant branch stars define the light profile of the system. In fact, an analysis by Hurley (2007) cautioned that the difference between mass and light distributions can lead to a large observed core to half-light radius ratio for GCs with single stars and binaries only.

Here we continue the search for indirect IMBH fingerprints by focusing on the consequences of the presence of an IMBH on mass segregation. Through direct $N$-body simulations we show that the presence of a large (of order $1 \%$ of the total mass) central mass significantly inhibits the process of mass segregation, even among only visible main-sequence stars and giants. To the best of our knowledge this effect was first briefly mentioned in Baumgardt et al. (2004) but was left without further quantitative analysis. Quenching of mass segregation is present in all of our simulations with an IMBH, independent of the initial conditions of the cluster, including variations in initial mass function, density profile, strength of the galactic tidal interaction, number of particles, and initial binary fraction. We find that a differential measurement of the average mass between the center and the half-light radius is effective in separating star clusters with and without an 
IMBH, provided that the stellar system is at least five initial halfmass relaxation times old. This measure is observationally feasible with current data (see, e.g., De Marchi et al. 2007 and references therein) and can lead to the selection of a promising set of IMBH host candidates. A direct observational application of this approach is left to a companion paper. Here we focus instead on building the theoretical framework for such analysis. In $\S 2$ we describe our numerical simulations, in $\S 3$ we discuss our results, and in $\S 4$ we present our conclusions.

\section{NUMERICAL SIMULATIONS}

The numerical simulations presented in this paper have been carried out with a state-of-the-art direct $N$-body code for star cluster dynamics, NBODY6 (Aarseth 2003). NBODY6 has been modified as discussed in Trenti et al. (2007a) to improve accuracy in the presence of an IMBH and uses regularization of close gravitational encounters without any softening. This makes it optimal to follow interactions within the sphere of influence of the IMBH.

\subsection{Units and Timescales}

NBODY6 uses the standard system of units of Heggie \& Mathieu (1986) in which $G=M=-4 E_{T}=1$, where $G$ is the gravitational constant, $M$ is the total mass, and $E_{T}$ is the total energy of the system. In this system of units, the half-mass relaxation time, which is the relevant timescale for mass segregation and energy equipartition, is defined as follows (Spitzer 1987):

$$
t_{r_{h}}=\frac{0.138 N r_{h}^{3 / 2}}{\ln (0.11 N)}
$$

where $N$ is the number of stars in the system and $r_{h}$ is the halfmass radius. In physical units $t_{r_{h}}$ can be expressed as (Djorgovski 1993):

$t_{r_{h}}=\frac{8.9 \cdot 10^{5} y r}{\ln (0.11 N)} \times\left(\frac{1 M_{\odot}}{\left\langle m_{*}\right\rangle}\right) \times\left(\frac{M}{1 M_{\odot}}\right)^{0.5} \times\left(\frac{r_{h}}{1 p c}\right)^{1.5}$,

where $\left\langle m_{*}\right\rangle$ is the average mass of a star.

\subsection{Initial Conditions}

Galactic GCs are made of some of the oldest stars in our galaxy (see, e.g., Krauss \& Chaboyer 2003) and are collisionally relaxed systems. Their two-body half-mass relaxation times, $t_{r_{h}}$, are shorter than their age (see, e.g., Heggie \& Hut 2003); thus their initial conditions are largely unknown. However, the evolution on a relaxation timescale is only weakly dependent on the initial configuration as the system evolves toward a self-similar configuration in which the density and light profiles are determined primarily by the efficiency of kinetic energy production in the core due to gravitational encounters (Vesperini \& Chernoff 1994; Trenti et al. 2007a, 2007b). In this paper, we explore a number of different initial configurations, varying the initial mass function, the initial density profile, the strength of the galactic tidal field, and the fraction of primordial binaries in order to verify that the evolution of the system is indeed independent of the initial configuration. The initial density profile is always that of a single-mass King (1966) model, but we use a full mass spectrum in the $N$-body calculations. The number of particles is varied from $N=8192$ to 32,768 to quantify the evolution of mass segregation with and without an IMBH. The details of our runs are reported in Table 1.
Initial stellar masses were drawn from either a Salpeter (1955) or Miller \& Scalo (1979) initial mass function (IMF); that is

$$
\xi(m) \propto m^{\alpha},
$$

with $\alpha=-2.35$ and $m \in[0.2: 100] M_{\odot}$ for the Salpeter IMF, while for the Miller \& Scalo IMF the power-law slope is the following: $\alpha=-1.25$ for $m \in[0.2: 1] M_{\odot}, \alpha=-2.0$ for $m \in$ $[1: 2] M_{\odot}, \alpha=-2.3$ for $m \in[2: 10] M_{\odot}$, and $\alpha=-3.3$ for $m \in[10: 100] M_{\odot}$. In addition, we have also carried out control runs that extend the IMF down to $0.1 M_{\odot}$, as such stars exist in GCs but are not bright enough to be detected in most of the cluster.

We handled stellar evolution by assuming a turnoff mass $M_{\mathrm{TO}}=0.8 M_{\odot}$, and instantaneously reducing all stars to their final state at the beginning of the simulation. Stars with masses $0.8 M_{\odot} \leq m<8.0 M_{\odot}$ were assumed to become white dwarfs and reduced to a final mass as prescribed in Hurley et al. (2000). Stars in the ranges 8.0-25.0 and 25.0-100.0 $M_{\odot}$ became neutron stars (unity retention fraction assumed) and black holes, and were reduced linearly to $1.3-2.0$ and 5-10 $M_{\odot}$, respectively. Our model makes the approximation that most of the relevant stellar evolution occurs on a timescale shorter than a relaxation time. This choice is appropriate to model the dynamics of an old GC on a relaxation timescale with only a limited number of particles and is more realistic than using an unevolved mass spectrum appropriate for young star clusters when $N \lesssim 30,000$ (Trenti et al. 2008).

In addition, about half of our runs included primordial binaries, an important component of many GCs (see, e.g., Pulone et al. 2003) that can influence the evolution of mass segregation. In fact, binaries are on average twice as massive as singles and thus tend to segregate in the core of the system (see, e.g., Heggie et al. 2006). We define the fraction of binaries to be

$$
f_{b}=\frac{n_{b}}{n_{s}+n_{b}}
$$

where $n_{s}$ and $n_{b}$ are the initial number of single stars and binaries, respectively. Thus, a run with $N=n_{s}+n_{b}=8192$ and $f_{b}=$ 0.1 actually has $8192+819=9011$ objects. As the dynamical influence of binaries tends to saturate for $f_{b} \approx 0.1$ (Vesperini \& Chernoff 1994; Heggie et al. 2006), all our runs with primordial binaries have $f_{b}=0.1$, a number similar to the observed binary fraction of many old GCs (e.g., Albrow et al. 2001). Binaries were initialized as in Heggie et al. (2006), that is, from a flat distribution in binding energy from $\epsilon_{\min }$ to $133 \epsilon_{\min }$, with $\epsilon_{\min }=$ $\left\langle m_{*}\right\rangle \sigma_{c}(0)$. Here, $\sigma_{c}(0)$ is the initial central velocity dispersion of the cluster.

To half of the simulations, we added an IMBH with mass $M_{\text {IMBH }} \approx 0.01(\approx 1 \%$ of the entire cluster $)$, the same ratio as a $\sim 10^{3} M_{\odot}$ black hole would have to a GC of mass $10^{5} M_{\odot}$. In some of the simulations (see Table 1), we increased the mass of the IMBH to study the dependence of mass segregation on this parameter.

All objects in our runs were treated as point masses, thus neglecting stellar evolution and collisions as well as any growth of the IMBH due to accretion of tidally disrupted stars. These effects have only a minor influence on the late-time dynamics of the cluster, since actual collisions are rare after massive stars have evolved, and accretion onto the IMBH is minimal (see, e.g., Baumgardt et al. 2004).

The evolution of the clusters includes the tidal force from the parent galaxy, assuming circular orbits with radii such that the 
TABLE 1

Summary of the $N$-Body Simulations

\begin{tabular}{|c|c|c|c|c|c|c|c|c|c|}
\hline Name & $N$ & $W_{0}$ & IMF & $M_{\mathrm{IMBH}} / M_{\mathrm{tot}}$ & $M_{\mathrm{IMBH}} / M_{\odot}$ & $f_{b}$ & $\langle\Delta\langle m\rangle\rangle$ & $\Delta\langle m\rangle_{\min }$ & $\Delta\langle m\rangle_{\max }$ \\
\hline 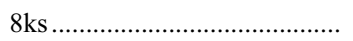 & 8192 & 7.0 & Sal & N/A & N/A & 0 & 0.07 & 0.040 & 0.112 \\
\hline $8 \mathrm{~km}$ & 8192 & 7.0 & $M \& S$ & $\mathrm{~N} / \mathrm{A}$ & N/A & 0 & 0.13 & 0.095 & 0.167 \\
\hline 8kbs5 & 8192 & 5.0 & Sal & $\mathrm{N} / \mathrm{A}$ & $\mathrm{N} / \mathrm{A}$ & 0.1 & 0.09 & 0.029 & 0.138 \\
\hline 8kbs & 8192 & 7.0 & Sal & N/A & N/A & 0.1 & 0.10 & 0.071 & 0.143 \\
\hline 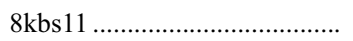 & 8192 & 11.0 & Sal & $\mathrm{N} / \mathrm{A}$ & $\mathrm{N} / \mathrm{A}$ & 0.1 & 0.09 & 0.037 & 0.116 \\
\hline 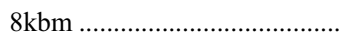 & 8192 & 7.0 & $M \& S$ & $\mathrm{~N} / \mathrm{A}$ & N/A & 0.1 & 0.09 & 0.048 & 0.130 \\
\hline 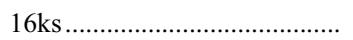 & 16,384 & 7.0 & Sal & $\mathrm{N} / \mathrm{A}$ & N/A & 0 & 0.11 & 0.071 & 0.158 \\
\hline 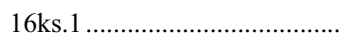 & 16,384 & 7.0 & Sal & $\mathrm{N} / \mathrm{A}$ & $\mathrm{N} / \mathrm{A}$ & 0 & 0.14 & 0.112 & 0.191 \\
\hline $16 \mathrm{~km}$ & 16,384 & 7.0 & M\&S & $\mathrm{N} / \mathrm{A}$ & N/A & 0 & 0.14 & 0.112 & 0.174 \\
\hline 16kbs & 16,384 & 7.0 & Sal & N/A & N/A & 0.1 & 0.09 & 0.060 & 0.140 \\
\hline $16 \mathrm{kbm}$ & 16,384 & 7.0 & $\mathrm{M} \& \mathrm{~S}$ & N/A & N/A & 0.1 & 0.10 & 0.067 & 0.127 \\
\hline $32 \mathrm{~km}$ & 32,768 & 7.0 & M\&S & N/A & N/A & 0 & 0.14 & 0.108 & 0.161 \\
\hline 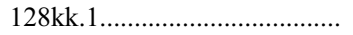 & 131,072 & 7.0 & Kroupa & N/A & N/A & 0 & $0.13^{*}$ & N/A & N/A \\
\hline $8 \mathrm{ksBH}$ & 8193 & 7.0 & Sal & 0.03 & 104.0 & 0 & 0.05 & 0.014 & 0.091 \\
\hline $8 \mathrm{kmbh}$ & 8193 & 7.0 & $\mathrm{M} \& \mathrm{~S}$ & 0.01 & 42.1 & 0 & 0.07 & 0.036 & 0.121 \\
\hline $8 \mathrm{kmBH}$ & 8193 & 7.0 & $M \& S$ & 0.03 & 129.3 & 0 & 0.06 & 0.011 & 0.097 \\
\hline 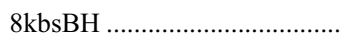 & 8193 & 7.0 & Sal & 0.03 & 114.4 & 0.1 & 0.04 & -0.014 & 0.080 \\
\hline 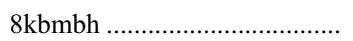 & 8193 & 7.0 & $M \& S$ & 0.015 & 69.5 & 0.1 & 0.04 & -0.021 & 0.079 \\
\hline $16 \mathrm{ksbh}$ & 16,385 & 7.0 & Sal & 0.015 & 103.1 & 0 & 0.05 & 0.023 & 0.090 \\
\hline 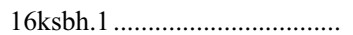 & 16,385 & 7.0 & Sal & 0.015 & 60.9 & 0 & 0.06 & 0.013 & 0.118 \\
\hline $16 \mathrm{kmbh}$ & 16,385 & 7.0 & $M \& S$ & 0.015 & 128.2 & 0 & 0.08 & 0.027 & 0.113 \\
\hline 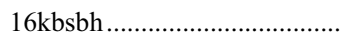 & 16,385 & 7.0 & Sal & 0.01 & 113.4 & 0.1 & 0.04 & 0.015 & 0.078 \\
\hline $16 \mathrm{kbmbh}$ & 16,385 & 7.0 & $\mathrm{M} \& \mathrm{~S}$ & 0.01 & 141.0 & 0.1 & 0.05 & 0.015 & 0.084 \\
\hline $32 \mathrm{kmbh}$ & 32,769 & 7.0 & M\&S & 0.01 & 240.0 & 0 & 0.07 & 0.051 & 0.101 \\
\hline 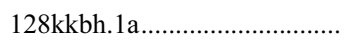 & 131,072 & 7.0 & Kroupa & 0.013 & 1000.0 & 0 & $0.09^{*}$ & $\mathrm{~N} / \mathrm{A}$ & $\mathrm{N} / \mathrm{A}$ \\
\hline 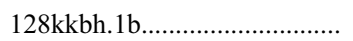 & 131,072 & 7.0 & Kroupa & 0.009 & 1000.0 & 0 & $0.06^{*}$ & N/A & N/A \\
\hline
\end{tabular}

Notes.-We calculated the average, maximum, and minimum values for $\Delta\langle m\rangle$ (in solar mass units) between 5 and 12 relaxation times for each run. Values with asterisks are not averages, but are from a single snapshot. The name of each run indicates: the number of stars in the simulation (8k, 16k, $32 \mathrm{k}$, or 128k); the presence of primordial binaries ( $b$ if $\left.f_{b}>0\right)$; the IMF ("m" for Miller \& Scalo [M\&S], "s" for Salpeter [Sal], and " $\mathrm{k}$ " for Kroupa); the presence of an IMBH (absent for none, "bh" for small BH mass, and "BH" for larger - see also the fifth column); the value of $W_{0}$ if different from 7.0 ; and the control run with IMF lower cutoff at $0.1 M_{\odot}$ (".1" suffix).

tidal cutoff radius is self-consistent with the value of the King parameter $W_{0}$ used. The galactic tidal field is treated as that due to a point mass, and the tidal force acting on each particle is computed using a linear approximation of the field. Particles that become unbound are removed from the system. For full details of the tidal field treatment, see Trenti et al. (2007b).

For validation purposes, we also analyzed a few snapshots from three runs with $N=131,072$ carried out by Baumgardt \& Makino (2003) and by Baumgardt et al. (2004) with and without a central IMBH, kindly made available by the authors. These runs include full stellar evolution using the Hurley et al. (2000) tracks, but no primordial binaries. In addition, accretion of tidally disrupted stars onto the IMBH is included. The initial star positions and velocities from these runs were also drawn from a King model with $W_{0}=7.0$, but instead the mass spectrum was drawn from a Kroupa (2001) IMF, with $\alpha=-1.3$ for $m \in$ $[0.1: 0.5] M_{\odot}$ and $\alpha=-2.3$ for $m>0.5 M_{\odot}$. The upper cutoff mass was $15 M_{\odot}$ for run $128 \mathrm{kk} .1,30 M_{\odot}$ for run $128 \mathrm{kkbh} .1 \mathrm{a}$, and $100 M_{\odot}$ for run $128 \mathrm{kkbh} .1 \mathrm{~b}$. These snapshots provide us with a control group against which we can test the validity of our own models and also allow us to probe the extrapolation of our results to higher $N$.

\section{RESULTS}

\subsection{Overall Evolution of the Star Clusters}

A star cluster with single stars only and no central IMBH evolves toward core collapse within a few relaxation times. The collapse is eventually halted when the central density is high enough to dynamically form binaries. At this stage gravothermal oscillations set in and the density profile of the cluster remains self-similar until the final stages of tidal dissolution of the system. The existence of either primordial binaries or an IMBH serves as an energy source to counteract the collapse, resulting in a more significant core (Trenti et al. 2007a). This is confirmed in all models (see, e.g., Fig. 1 for the evolution of the core and halfmass radius in our $32 \mathrm{k}$ simulations). Here, we use the Casertano $\&$ Hut (1985) definition of the core radius, namely

$$
r_{c}=\frac{\sum_{i=1}^{N} r_{i} \rho_{i} m_{i}}{\sum_{i=1}^{N} \rho_{i} m_{i}},
$$

where $m_{i}$ is the mass of the $i$ th star, $r_{i}$ is its radius from the cluster Q3 center of mass, and the density $\rho_{i}$ around each particle is calculated using the distance to the fifth-closest neighbor.

As expected from previous investigations based on equal-mass particles (Trenti et al. 2007b), the density profile of our clusters progresses to a self-similar configuration, which is independent of the initial configuration of the stars and the IMF. This independence justifies our treatment of stellar evolution at the beginning of the simulations and provides further evidence of the erasure of initial conditions after a few relaxation times. The overall evolution of star clusters with and without an IMBH and/or primordial binaries has been discussed in the literature (Baumgardt \& Makino 2003; Baumgardt et al. 2004; Heggie et al. 2006; Trenti et al. 2007a, 2007b; Fregeau \& Rasio 2007; Hurley 2007). Here we focus instead on a novel aspect that has a promising observational signature, namely the evolution of mass segregation for runs with an IMBH. 


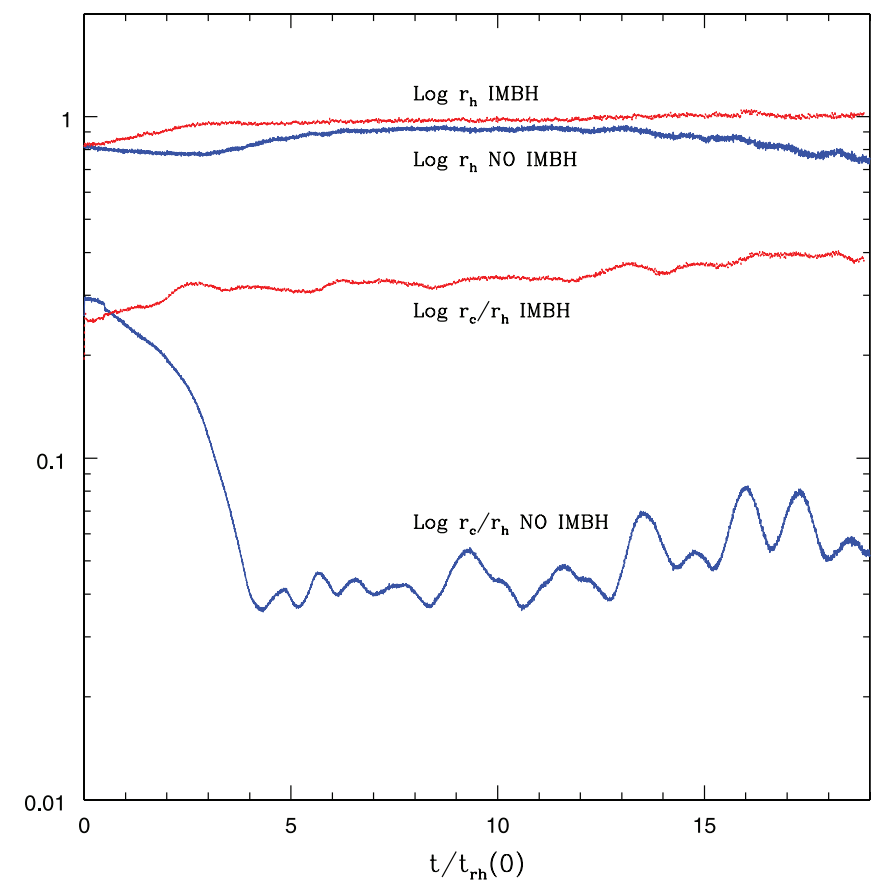

FIG. 1.-Evolution of the three-dimensional half-mass radius $\left(r_{h}\right)$ and of the core to half-mass radius ratio $\left(r_{c} / r_{h}\right)$ in $N$-body units for our $N=32,769$ simulations with (red curves) and without (blue curves) an IMBH. The presence of an IMBH prevents core collapse. We have smoothed the curves by applying a triangular smoothing window of size $1.0 t_{r_{h}}(0)$.

\subsection{Mass Segregation}

As our overarching goal is to propose a viable observational test to identify a star cluster that is likely to harbor an IMBH, we took steps throughout our analysis to replicate observational data as closely as possible. We limited our analysis to data projected onto two dimensions and excluded stellar remnants from our calculations of observationally accessible quantities. Most of our runs did not have stars with masses below $0.2 M_{\odot}$, because these are generally too dim to detect with a high completeness. However, we did perform two $16 \mathrm{k}$ control runs with masses down to $0.1 M_{\odot}$ to ensure that the presence of smaller, undetectable stars did not affect the mass segregation. For a proper comparison to the other runs, we did exclude the stars with masses between 0.1 and $0.2 M_{\odot}$ from the calculation of the observationally accessible quantities for these runs.

Binary systems were handled by including only the brighter member in the analysis of the observationally accessible quantities. This choice is motivated by the fact that for real observations, masses will need to be estimated from luminosities. Since binaries in GCs are typically not resolved (the separations are below a few astronomical units for the range of binding energies considered), we observe mainly single sources. Because the luminosity of a main-sequence star is highly sensitive to its mass $\left(L \sim M^{3.5}\right)$, the lighter star contributes very little to the overall luminosity in many cases, and thus the total luminosity will be very similar to that of the brighter member.

To quantify the effects of mass segregation, we examined the radial variations in average stellar mass - or equivalently-in the slope of the mass function (if the mass function is a power law in the mass range considered, then there is a one-to-one relation between the average mass and the slope). As a consequence of energy equipartition, heavier particles sink to the center of the cluster within a few relaxation times, increasing the difference in average mass between the center and the halo of the cluster. As our main diagnostic of mass segregation, we define

$$
\Delta\langle m\rangle=\langle m\rangle(r=0)-\langle m\rangle\left(r=r_{h}\right)
$$

where $r_{h}$ is the projected half-mass radius of the cluster (computed using only visible stars), and $\langle m\rangle$ is the average mass for Q4 main-sequence stars with $m \in[0.2: 0.8] M_{\odot}$. Both of these measurements are taken from projected radial bins, each containing $5 \%$ of the cluster's visible stars. Because nearly all of the deviation in $\langle m\rangle(r)$ occurs within this radial range, we are maximizing our baseline for measuring mass segregation while using fields with a reasonable number of stars. This definition also allows for a straightforward comparison to observational data, as only two fields per cluster are sufficient.

Figure 2 depicts the evolution of $\Delta\langle m\rangle$ for our $N=16,384$ to 32,768 runs without primordial binaries. For each run, we analyze the configuration of the system every $15 \mathrm{~N}$-body units (which corresponds to more than 10 measurements per relaxation time). Runs with an IMBH are represented as red points, while runs without are blue points. Because they were drawn from single-mass King models, our clusters begin out of equilibrium. On a relaxation timescale, we see them evolve toward a new quasi-equilibrium state. After $\sim 5 t_{r_{h}}(0)$, most clusters have settled into this equilibrium, with those harboring an IMBH showing a smaller amount of mass segregation, that is, smaller values of $\Delta\langle m\rangle$. The points from the control snapshots $128 \mathrm{kk} .1$, $128 \mathrm{kkbh} .1 \mathrm{a}$, and $128 \mathrm{kkbh} .1 \mathrm{~b}$, as well as the two $16 \mathrm{k}$ control runs (16ks. 1 and 16ksbh.1) are also plotted in Figure 2 and are in good agreement with those from our models. The data in Figure 2 come from a variety of initial configurations, not only in terms of the particle number but also in terms of IMF. The use of a differential indicator for mass segregation allows us to cancel out the dependence on the global value of $\langle m\rangle$ (or on the global mass function slope).

Simulations with a Salpeter IMF (16ks, 16ksbh) contain many more massive remnants than the number allowed by a Miller \& Scalo IMF (16km, 16kmbh, $32 \mathrm{~km}, 32 \mathrm{kmbh}$ ). For example, a $16 \mathrm{k}$ simulation with a Salpeter IMF and $m \in[0.2: 100] M_{\odot}$ initially contains $\sim 20$ stellar mass black holes, whereas a Miller \& Scalo IMF will only have $\sim 1$. This difference causes us to observe a slower growth of $\Delta\langle m\rangle$ in the Salpeter IMF runs, as a central cluster of stellar mass black holes partially quenches mass segregation of visible stars, much like an IMBH. However, stellar mass black holes eject one another from the system within a few relaxation times (see also Merritt et al. 2004), so eventually these systems fully develop the amount of mass segregation observed in runs starting from a Miller \& Scalo IMF.

The control runs also reflect this trend. The $128 \mathrm{k}$ run without an IMBH (128kk.1) has a maximum allowed IMF mass of $15 M_{\odot}$, meaning there are essentially no massive remnants. After five relaxation times, it is in good agreement with our simulations drawn from a Miller \& Scalo IMF, which also produces very few massive remnants. The runs with an IMBH $(128 \mathrm{kkbh} .1 \mathrm{a}$ and $128 \mathrm{kkbh} .1 \mathrm{~b}$ ), which have maximum initial masses of 30 and $100 M_{\odot}$, respectively, are both consistent with our other runs with an IMBH. However, 128kkbh.1a's lower maximum mass results in fewer massive remnants, and thus a value for $\Delta\langle m\rangle$ closer to $16 \mathrm{kmbh}$, which also contains few stellar mass black holes. $128 \mathrm{kkbh} .1 \mathrm{~b}$ has more massive remnants and behaves similarly to $16 \mathrm{ksbh}$, as we would expect. Finally, we see that our $16 \mathrm{k}$ control runs with a lower IMF cutoff at $0.1 M_{\odot}$ fall somewhere between the Miller \& Scalo runs and the Salpeter runs as far as mass segregation is concerned. Although we draw from a Salpeter 


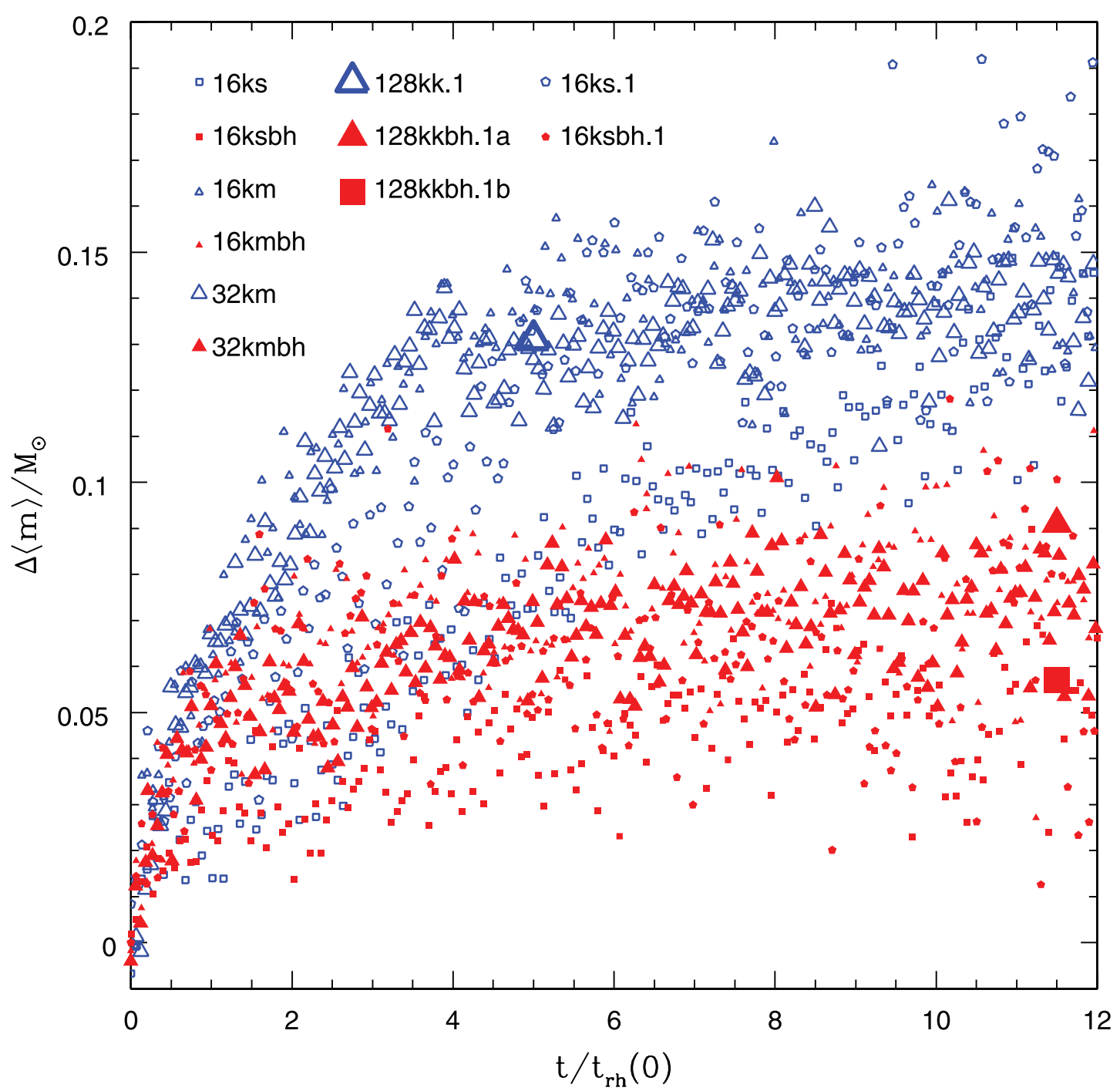

FIG. 2.-Evolution of mass segregation (via $\Delta\langle m\rangle$, expressed in $M_{\odot}$ ) across the span of all $N$-body simulations with $N \geq 16,384$ and $f_{b}=0$. Red points are from simulations with an IMBH, while blue points represent runs with no massive central object. The runs have no primordial mass segregation $(\Delta\langle m\rangle=0)$, but on a relaxation timescale, the systems settle to a quasi-equilibrium configuration with varying degrees of mass segregation. A central IMBH quenches the mass segregation and keeps $\Delta\langle m\rangle \lesssim 0.09 M_{\odot}$.

IMF in the control runs, the lower minimum IMF mass in these simulations results in fewer massive remnants than the other Salpeter runs $(\sim 8$ stellar mass black holes instead of $\sim 20)$, but more than a Miller \& Scalo IMF would produce. We also note the increased scatter in these two runs as a result of excluding the large number of main-sequence stars with masses $0.1 M_{\odot}<$ $m<0.2 M_{\odot}$ in the calculation of the observationally accessible quantities.

The situation is very similar when primordial binaries are included (see Fig. 3): runs with and without an IMBH again become well separated after $\sim 5 t_{r_{h}}(0)$. As expected, primordial binaries carry lighter particles toward the center of the cluster (e.g., a $0.6+0.2 M_{\odot}$ binary will sink to the center like a $0.8 M_{\odot}$ single star, but will be observed as a single unresolved source with the approximate luminosity of a $0.6 M_{\odot}$ star). Hence, mass segregation is partially suppressed when compared to the runs where $f_{b}=0$. This difference in $\Delta\langle m\rangle$ is more significant in the runs with a Miller \& Scalo IMF as compared to runs with a Salpeter IMF. Because the runs drawn from a Miller \& Scalo IMF lack massive remnants, binary stars become more gravitationally dominant and therefore have a more significant impact on the dynamics. Fortunately, the binary-driven quenching of mass segregation is weak when compared to IMBH-driven quenching and thus it is possible to discriminate between systems with and without an IMBH on the basis of $\Delta\langle m\rangle$, without the need of assuming a binary fraction.

Combining the data from all our simulations with and without binaries, we can identify three regions for the value of $\Delta\langle m\rangle$ in a collisionally relaxed GC, irrespective of its binary fraction:

$\Delta\langle m\rangle \gtrsim 0.1 M_{\odot}$.- The system is unlikely to contain a central IMBH.

$\Delta\langle m\rangle \lesssim 0.07 M_{\odot} \cdot$ - The system is a good candidate to harbor an IMBH.

$0.07 M_{\odot} \lesssim \Delta\langle m\rangle \lesssim 0.1 M_{\odot}$. - The system may or may not contain an IMBH, depending on its binary fraction and on the global IMF (and in particular on the number of massive dark remnants).

In addition, an estimate of the binary fraction based on the presence of a parallel main sequence in the color-magnitude diagram is possible for many observed clusters (Milone et al. 2008). Application of the mass segregation diagnostic therefore can 


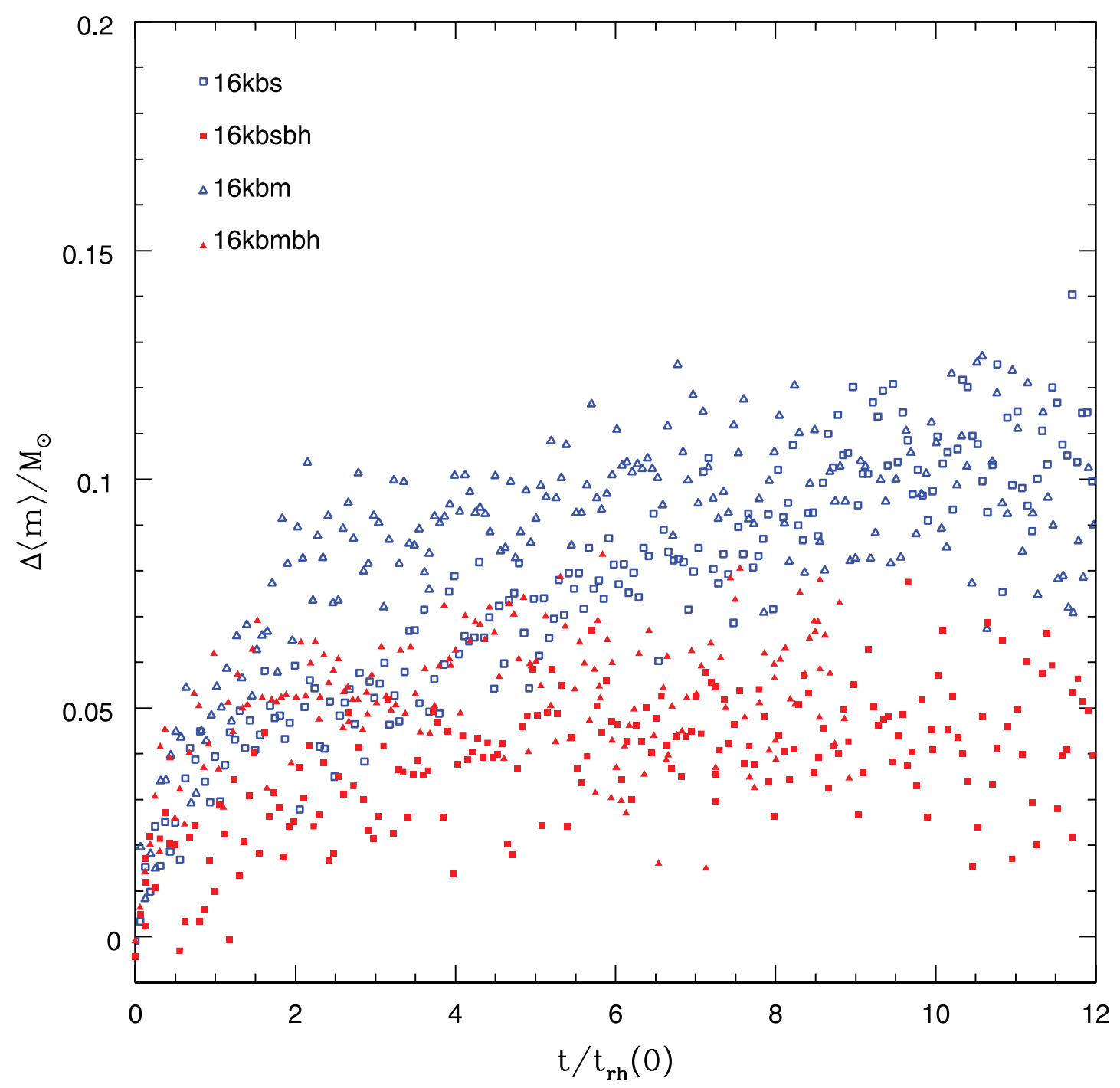

Fig. 3.-Evolution of mass segregation as in Fig. 1, but for our $N \geq 16,384$ simulations with primordial binaries. Qualitatively, we see that the results are similar to those of the runs with single stars, but the equilibrium values of $\Delta\langle m\rangle$ are marginally lower at later times when compared to those where $f_{b}=0$.

account for the actual number of binaries, resulting in a further reduction in the size of the region of uncertainty.

Including the set of runs with $N=8192$, not shown in the plots but whose $\Delta\langle m\rangle$ is reported in Table 1, we see no trends in $\Delta\langle m\rangle$ caused by an increase in the number of objects in a cluster up to $N=32,768$. In addition, the $N=131,072$ control snapshots are consistent with our results, strengthening the independence in the evolution of $\Delta\langle m\rangle$. An increase in the number of particles reduces the deviation from snapshot to snapshot. This actually improves the application of this indicator to actual GCs, where the number of stars is significantly larger than in our runs. Similarly, we see no significant trends in $\Delta\langle m\rangle$ caused by increasing the IMBH mass up to $M_{\mathrm{IMBH}}=0.03$, that is, $3 \%$ of the entire cluster (see Table 1). This suggests that reducing $M_{\mathrm{IMBH}}$ below 0.01 would still result in a quenching of mass segregation.

\subsection{The Origin of IMBH-induced Quenching of Mass Segregation}

The onset of mass segregation along with the initial contraction of the cluster brings the most massive stars and remnants into a dense environment. Even in clusters with only single stars, the dynamical formation of binaries is inevitable. Because $M_{\mathrm{IMBH}}$ is much larger than the typical stellar mass, the IMBH has an extremely high probability of exchanging into a binary in a close three-body encounter. It therefore spends much of its lifetime in a binary or stable, higher $N$ system (in more than $90 \%$ of our snapshots the IMBH is a member of a multiple system). As a result, when massive main-sequence stars in our simulations sink to the core after energy exchanges with other stars, they are efficiently "heated up" and scattered away from the core in encounters with the IMBH and any companions it has. The IMBH stochastically moves around the core as a result of these encounters and, this further enhances the interaction rate because the scatter cone is continuously replenished. This mechanism for quenching mass segregation naturally explains the lack of dependence of $\Delta\langle m\rangle$ on the number of particles used and the minimal dependence on $M_{\mathrm{IMBH}}$, as well as suggesting an additional explanation as to why the presence of primordial binaries further reduces mass segregation.

\section{DISCUSSION AND CONCLUSIONS}

We have carried out a large set of direct $N$-body simulations of star clusters with and without an IMBH, including a realistic mass spectrum and primordial binaries. While previous research has focused its attention mainly on the effects of an IMBH on the surface brightness and velocity dispersion profiles of the 
clusters - signatures that are difficult to observe-we searched instead for a different fingerprint of the presence of an IMBH. The existence of a massive, central object quenches mass segregation, and this effect manifests itself in collisionally relaxed clusters through decreased radial variation in the average mass of main-sequence stars. This effect does not depend on the mass of the black hole as long as it is dominant over the typical mass of a star, nor on the details of the initial configuration of the system such as initial mass function, density profile, and tidal field strength. The amount of mass segregation is only weakly dependent on the binary fraction of the cluster. This result allows us to use the amount of mass segregation to separate collisionally relaxed clusters with and without an IMBH without the need of additional modeling assumptions.

A critical requirement for the proposed signature is that the system be well relaxed, so that it has already attained equilibrium with respect to mass segregation. From our simulations it turns out that this takes about $5 t_{r_{h}}(0)$. However, we can only observe the current half-mass relaxation time, and this might be shorter than its initial value if the system has lost a large fraction of its original mass. To compare our simulations to observations, we must thus conservatively restrict ourselves to GCs that:

1. Are not too influenced by the galactic tidal field (i.e.,, with a tidal to half-light radius $r_{t} / r_{\mathrm{hl}} \gtrsim 10$, which corresponds to tidal fields weaker than the weakest field in our simulations);

2 . Have half-mass (three-dimensional) relaxation times below $\approx 1.5 \mathrm{Gyr}$, that is, an age above $8 t_{r_{h}}$. This leaves room for a mass loss of about $50 \%$ of the initial mass while still giving an integrated age of about $5 t_{r_{h}}$. In terms of observable quantities, this translates into a half-light relaxation time below $\approx 1$ Gyr.

Based on the Harris (1996) catalog, 31 galactic GCs satisfy these stringent requirements in terms of relaxation time and $r_{t} / r_{\mathrm{hl}}$. The proposed diagnostic could probably be applied to more clusters after properly evaluating a dynamical model for their configuration and eventually accepting some uncertainty in the selection of likely candidates to harbor an IMBH.

Thanks to the HST treasury survey of galactic GCs, data exist for the cores of many clusters that explore deep enough to see main-sequence stars down to around $0.2 M_{\odot}$. Along the same lines, De Marchi et al. (2007), among others, have also acquired images of clusters around the half-light radius in order to calculate the global mass function of the system. The existing data from De Marchi et al. (2007) are sufficient to apply this diagnostic to a few actual clusters, and the results from such a comparison will be presented in a companion paper.

In closing, we stress again that while the amount of mass segregation has been proven here to be a viable indicator for the presence of an IMBH in simulated star clusters, we cannot use this method alone to claim the detection of an IMBH. However, by combining the measure of mass segregation with all other constraints from the velocity dispersion and surface brightness profiles, we can select the clusters that seem most likely to harbor an IMBH while at the same time excluding some others from further scrutiny. Once we have identified those clusters that are most promising, future observations, such as proper-motion studies, can focus their efforts to secure a robust detection.

We thank Enrico Vesperini for useful discussions and suggestions, Holger Baumgardt for sharing some of his data with us, and the referee for a careful reading of the manuscript and for constructive suggestions. This work was partially supported by NASA grant HST-AR11284.

\section{REFERENCES}

Aarseth, S. 2003, Gravitational N-Body Simulations. (Cambridge: Cambridge Univ. Press)

Albrow, M. D., Gilliland, R. L., Brown, T. M., Edmonds, P. D., Guhathakurta, P., \& Sarajedini, A. 2001, ApJ, 559, 1060

Bahcall, J. N., \& Wolf, R. A. 1976, ApJ, 209, 214

Baumgardt, H., Hut, P., Makino, J., McMillan, S., \& Portegies Zwart, S. 2003a, ApJ, 582, L21

Baumgardt, H., \& Makino, J. 2003, MNRAS, 340, 227

Baumgardt, H., Makino, J., \& Ebisuzaki, T. 2004, ApJ, 613, 1143

Baumgardt, H., Makino, J., Hut, P., McMillan, S., \& Portegies Zwart, S. 2003b, ApJ, 589, L25

Casertano, S., \& Hut, P. 1985, ApJ, 298, 80

De Marchi, G., Paresce, F., \& Pulone, L. 2007, ApJ, 656, L65

Djorgovski, S. 1993, in ASP Conf. Ser. 50, Structure and Dynamics of Globular Clusters, ed. S. G. Djorgovski \& G. Meylan (San Francisco: ASP), 373

Dull, J. D., Cohn, H. N., Lugger, P. M., Murphy, B. W., Seitzer, P. O., Callanan, P. J., Rutten, R. G. M., \& Charles, P. A. 2003, ApJ, 585, 598

Fregeau, J., \& Rasio, F. 2007, ApJ, 658, 1047

Gebhardt, K., Rich, R. M., \& Ho, L. C. 2002, ApJ, 578, L41

. 2005, ApJ, 634, 1093

Gerssen, J., van der Marel, R. P., Gebhardt, K., Guhathakurta, P., Peterson, R. C., \& Pryor, C. 2002, AJ, 124, 3270 2003, AJ, 125, 376

Harris, W. E. 1996, AJ, 112, 1487

Heggie, D., \& Hut, P. 2003, The Gravitational Million-Body Problem (Cambridge: Cambridge Univ. Press)

Heggie, D. C., Hut, P., Mineshige, S., Makino, J., \& Baumgardt, H. 2007, PASJ, 59, L11
Heggie, D. C., \& Mathieu, R. D. 1986, in The Use of Supercomputers in Stellar Dynamics (Berlin: Springer), 233

Heggie, D. C., Trenti, M., \& Hut, P. 2006, MNRAS, 368, 677

Hurley, J. R. 2007, MNRAS, 379, 93

Hurley, J. R., Pols, O. R., \& Tout, C. A. 2000, MNRAS, 315, 543

King, I. R. 1966, AJ, 71, 64

Krauss, L. M., \& Chaboyer, B. 2003, Science, 299, 65

Kroupa, P. 2001, MNRAS, 322, 231

Makino, J., Fukushige, T., Koga, M., \& Namura, K. 2003, PASJ, 55, 1163

Merritt, D., Piatek, S., Portegies Zwart, S., \& Hemsendorf, M. 2004, ApJ, 608, L25

Miller, G. E., \& Scalo, J. M. 1979, ApJS, 41, 513

Miller, M. C., \& Colbert, E. J. M. 2004, Int. J. Mod. Phys. D, 13, 1

Milone, A. P., Piotto, G., Bedin, L. R., \& Sarajedini, A. 2008, Mem. Soc. Astron. Italiana, 79, 623

Noyola, E., Gebhardt, K., \& Bergman, M. 2008, ApJ, 676, 1008

Portegies Zwart, S. F., Baumgardt, H., Hut, P., Makino, J., \& McMillan, S. L. W. 2004, Nature, 428, 724

Pulone, L., De Marchi, G., Covino, S., \& Paresce, F. 2003, A\&A, 399, 121

Salpeter, E. E. 1955, ApJ, 121, 161

Spitzer, L. 1987, Dynamical Evolution of Globular Clusters (Princeton: Princeton Univ. Press)

Trenti, M., Ardi, E., Mineshige, S., \& Hut, P. 2007a, MNRAS, 374, 857

Trenti, M., Heggie, D. C., \& Hut, P. 2007b, MNRAS, 374, 344

Trenti, M., Ransom, S., Hup, P., \& Heggie, D. C. 2008, MNRAS, 387, 815

van der Marel, R. P. 2004, in Coevolution of Black Holes and Galaxies, ed.

L. C. Ho (Cambridge: Cambridge Univ. Press), 37

Vesperini, E., \& Chernoff, D. F. 1994, ApJ, 431, 231 


\section{AUTHOR QUERIES}

Q1 Au: Your article has been edited for grammar, consistency, and to conform to journal style (see Instructions to Authors). To expedite publication, we generally do not query every routine grammatical and style change made to a manuscript, although all substantive changes have been noted. Please review the article carefully. Note that we may be unable to make changes that conflict with journal style, obscure meaning, or create grammatical or other problems. Also note that article proofs via PDF do not show corrections that will have been made by the typesetter's proofreader, which generally include corrections of errors of page layout, figure placement, and spacing and font mistakes; feel free to mark any errors you notice in these areas. When sending us your corrections, if you are writing them by hand, please print clearly. If sending a fax, please do not write too close to the margins of the page, as these are often cut off in fax transmission. Finally, please note that a delayed, incomplete, or illegible response may delay the publication of your article while we contact you.

Q2 Au: ApJ uses "Galactic" for Milky Way and "galactic" otherwise. Please make sure these have been styled correctly throughout.

Q3 Au: In the sentence that includes equation (5), we have added the word "radius"; is this correct?

Q4 Au: Replaced " $r_{\mathrm{hm}}$ with " $r_{h}$ (half-mass radius), as previously defined in eq. (1); OK?

Q5 Au: Bahcall \& Wolf: Not cited in the text; please let us know where it should be cited, or omit from reference list.

Q6 Au: Makino et al. 2003: Not cited in the text; please let us know where it should be cited, or omit from reference list. 